\title{
TTR
}

Traduction, terminologie, rédaction

Jean-Marc Gouanvic. Hard-boiled fiction et Série noire : les

métamorphoses du roman policier anglo-américain en

français (1945-1960). Paris, Classiques Garnier, 2018, 281 p.

\section{Patricia Godbout}

Volume 32, numéro 2, 2e semestre 2019

URI : https://id.erudit.org/iderudit/1068913ar

DOI : https://doi.org/10.7202/1068913ar

Aller au sommaire du numéro

Éditeur(s)

Association canadienne de traductologie

ISSN

0835-8443 (imprimé)

1708-2188 (numérique)

Découvrir la revue

Citer ce compte rendu

Godbout, P. (2019). Compte rendu de [Jean-Marc Gouanvic. Hard-boiled fiction et Série noire : les métamorphoses du roman policier anglo-américain en français (1945-1960). Paris, Classiques Garnier, 2018, 281 p.] TTR, 32(2), 255-257.

https://doi.org/10.7202/1068913ar d'utilisation que vous pouvez consulter en ligne.

https://apropos.erudit.org/fr/usagers/politique-dutilisation/ 
German-speaking researchers), and for the exercises, it seems to me that language teachers would need to be present. The authors say (p. 93) that librarians "may" want to collaborate with such teachers, but I suspect it would be a necessity: how many librarians can provide instruction in editing (e.g., assist German speakers having difficulty rewriting German compound nouns in an MT-friendly way)?

The book struck me as both very interesting and very well written, but then I am not a member of any of its intended audiences. It will be of special interest to non-native readers and writers of English, including Translation Studies scholars. Also, given the authors' view that MT literacy training should be given by librarians, the reception of the book in library science journals will be crucial to the fulfilment of its purpose.

\section{References}

Bowker, Lynne and Jairo Buitrago Ciro (2015). "Investigating the usefulness of machine translation for newcomers at the public library." Translation and Interpreting Studies, 10, 2, pp. 165-186.

Bowker, Lynne and Jairo Buitrago Ciro (2018). "Localizing websites using machine translation. Exploring the connection between user experience and translatability." In S. Chan, ed. The Human Factor in Machine Translation. London, Routledge, pp. 138-151.

\section{Brian Mossop York University, ToRONTO}

Jean-Marc Gouanvic. Hard-boiled fiction et Série noire: les métamorphoses du roman policier anglo-américain en français (19451960). Paris, Classiques Garnier, 2018, 281 p.

Cet ouvrage s'inscrit dans la foulée de plusieurs autres publiés par Jean-Marc Gouanvic : Sociologie de la traduction (1999), sur la sciencefiction américaine dans l'espace culturel français des années 1950; Pratique sociale de la traduction (2007), qui présente une étude du roman réaliste américain dans le champ littéraire français entre 1920 et 1960; et Sociologie de l'adaptation et de la traduction (2014), qui se penche sur le roman d'aventures anglo-américain dans l'espace littéraire français pour les jeunes jusqu'en 1960. Dans tous ces ouvrages, Gouanvic applique les théories sociologiques de Pierre Bourdieu à l'étude de corpus de livres traduits. 
La période étudiée dans le présent ouvrage (1945-1960) semble être la période de prédilection de ce chercheur. Gouanvic s'intéresse ici à la collection «Série noire » de Gallimard, dirigée par Marcel Duhamel et, plus particulièrement, aux traductions françaises qui y sont publiées de romans de trois auteurs américains, Dashiell Hammett, Raymond Chandler et Chester Himes. Après avoir rappelé la place subalterne du roman policier dans l'espace littéraire durant cette période, l'auteur explique ce qui différencie les romans à énigme à la britannique, où il s'agit de démasquer un criminel, des romans de durs à cuire américains, romans noirs d'un autre genre dans lesquels la culpabilité plus diffuse s'étend à la société tout entière sur fond de puritanisme ambiant.

Cet ouvrage se penche principalement sur les mutations des livres de cette collection en traduction. L'auteur ne néglige pas pour autant le contexte éditorial qui les voit naître. À cet égard, il fait une utilisation intéressante de matériel d'archive pour établir entre autres l'babitus et la pratique éditoriale de Duhamel. Gouanvic montre que le fait de regrouper en collection des livres qui sont au point de départ éparpillés au sein de la culture source a pour effet d'en augmenter l'effet sur le lectorat, à la manière d'une loupe qui concentre les rayons du soleil.

Les transformations subies par les romans hard-boiled traduits pour la "Série noire» sont scrutées à la loupe, justement, dans le corpus retenu. Gouanvic abonde dans le sens de Benoît Tadié (2006) pour dire que le substrat puritain du roman policier hard-boiled tend à s'estomper en traduction française. Cet estompement ne tiendrait pas aux nombreux abrégements des textes sources, mais découlerait plutôt de la façon différente dont la culpabilité générale imprégnant les romans en langue originale est ressentie dans la société cible. Ainsi, ce ne serait pas du tout par puritanisme que les textes sources auraient subi des coupures, celles-ci étant plutôt motivées par le souci d'uniformiser le format des titres de la collection. Gouanvic souligne en outre que la dimension ludique de ces récits est accentuée dans les traductions, notamment par l'emploi du langage argotique, ce qui s'accorde avec leur finalité première qui est le divertissement des lecteurs.

Gouanvic fait état rapidement de critiques formulées depuis les années 1980 à l'endroit de la collection «Série noire», qui aurait manipulé les textes et donné une image franco-française des romans hard-boiled américains. Ces critiques se situent, selon lui, dans la droite ligne de la doxa traductologique tournée vers le texte source. 
Exprimant son désaccord avec l'affirmation de Clem Robyns (1990) selon laquelle ces traductions s'inséreraient dans la vieille tradition française des Belles Infidèles, Gouanvic insiste pour sa part sur le décalage inhérent à la traduction par rapport à la culture source et sur la réinterprétation obligée des formes et des idées. Ainsi, tout le travail de ce chercheur consistant à faire état des métamorphoses pas toujours heureuses que subissent les textes sources en traduction n'a pas pour objet de prendre les traducteurs en faute, mais bien plutôt d'examiner ce qu'il advient, dans le passage du texte source au texte cible, de l'illusio - c'est-à-dire du mécanisme d'adhésion aux normes et valeurs régissant le champ d'appartenance. Cet examen inclut l'étude comparative de traductions françaises parues dans les années 1930 et de leurs retraductions dans la «Série noire».

Bien que cela sorte évidemment du cadre de son étude, il eût été approprié, me semble-t-il, que Gouanvic fasse mention - dans son avant-propos, par exemple - des retraductions récentes qui ont été faites des romans de Hammett, Chandler et Himes (lesquelles ne figurent pas dans sa bibliographie). Celles-ci ont fourni à plusieurs commentateurs l'occasion de souligner, exemples à l'appui, les tares des traductions précédentes publiées dans la collection dirigée par Duhamel. Une telle mention aurait eu l'avantage de relier la présente étude de Gouanvic à une certaine actualité littéraire, d'autant que les trois auteurs sur lesquels il se concentre dans son ouvrage sont encore lus en français de nos jours.

\section{Références}

Gouanvic, Jean-Marc (1999). Sociologie de la traduction: la science-fiction américaine dans l'espace culturel français des années 1950. Arras, Artois Presses Université.

Gouanvic, Jean-Marc (2007). Pratique sociale de la traduction: le roman réaliste américain dans le champ littéraire français (1920-1960). Arras, Artois Presses Université.

Gouanvic, Jean-Marc (2014). Sociologie de l'adaptation et de la traduction: le roman d'aventures anglo-américain dans l'espace littéraire français pour les jeunes (1826-1960). Paris, Honoré Champion.

Robyns, Clem (1990). «The Normative Model of the Twentieth-Century Belles Infidèles: Detective Novels in French Translation». Target, 2, 1, p. 23-42.

Tadié, Benoît (2006). Le polar américain : la modernité et le mal. Paris, Presses universitaires de France.

Patricia Godbout Université de Sherbrooke 